\title{
ON S. GRIVAUX' EXAMPLE OF A HYPERCYCLIC RANK ONE PERTURBATION OF A UNITARY OPERATOR.
}

\author{
ANTON BARANOV, ANDREI LISHANSKII
}

\begin{abstract}
Recently, Sophie Grivaux showed that there exists a rank one perturbation of a unitary operator in a Hilbert space which is hypercyclic. We give a similar construction using a functional model for rank one perturbations of singular unitary operators.
\end{abstract}

\section{INTRODUCTION}

A continuous linear operator $T$ in a Fréchet space $F$ is said to be hypercyclic if there exists a vector $f \in F$ such that its orbit $\left\{T^{n} f\right\}_{n=0}^{\infty}$ is dense in $F$. In this case the vector $f$ is said to be hypercyclic for $T$. First examples of hypercyclic operator go back to G.D. Birkhoff, G.R. McLane, S. Rolewicz, while a systematic study of hypercyclicity phenomenon started in 1980-s with the thesis by C. Kitai and works by R.M. Gethner, G. Godefroy and J.H. Shapiro [5, 6]. We refer to the recent monographs [2, 9] for a detailed account of the theory.

Clearly, the identity operator is one of the most "nonhypercyclic" operators. However, already in 1991 K.C. Chan and J.H. Shapiro 3 showed that there exists hypercyclic operators in a Hilbert space of the form $I+K$, where the compact operator $K$ may belong to any Schatten class. It is clear that $I+R$ can not be hypercyclic when $R$ is a finite rank operator. Still, if we replace $I$ by a unitary operator, hypercyclicity is possible. In $2010 \mathrm{~S}$. Shkarin [14 produced an example of a unitary operator $U$ such that $U+R$ is hypercyclic for some rank two operator $R$. Shkarin asked whether $R$ can be taken to be of rank one. A positive answer was given by S. Grivaux [8]:

Theorem 1.1 (S. Grivaux, [8]). There exists a unitary operator $U$ in the space $\ell^{2}$ and a rank one operator $R$ such that $U+R$ is hypercyclic.

The proof of this theorem is based on an ingenious elementary construction involving a certain convergent inductive process as well as on the following sufficient condition for hypercyclicity also obtained by Grivaux [7. This result says that the operator is hypercyclic if there is a certain "continuous" family of eigenvectors with unimodular eigenvalues.

Theorem 1.2 (S. Grivaux, [7]). Let $X$ be a complex separable infinite-dimensional Banach space, and let $T$ be a bounded operator on $X$. Suppose that there exists a sequence $\left\{u_{n}\right\}_{n \geq 1}$ of vectors in $X$ having the following properties:

(i) $u_{n}$ is an eigenvector of $T$ associated to an eigenvalue $\lambda_{n}$ of $T$, with $\left|\lambda_{n}\right|=1$ and $\lambda_{n}$ are all distinct;

(ii) $\operatorname{span}\left\{u_{n}: n \geq 1\right\}$ is dense in $X$;

The authors were supported by the Chebyshev Laboratory (St. Petersburg State University) under RF Government grant 11.G34.31.0026 and by JSC "Gazprom Neft". 
(iii) for any $n \geq 1$ and any $\varepsilon>0$, there exists $m \neq n$ such that $\left\|u_{n}-u_{m}\right\|<\varepsilon$. Then $T$ is hypercyclic and even frequently hypercyclic.

The aim of the present paper is to give a proof of Theorem 1.1 by function theory methods. Our approach is based on a functional model for rank one perturbations of singular unitary operators. This model essentially goes back to a paper by V.V. Kapustin [10]. In the form that we will use, this model appeared in [1, Theorem 0.6] in the context of rank one perturbations of selfadjoint operators. This model translates any rank one perturbation of a unitary operator to some concrete operator in a space of analytic functions in the unit disk which is known as a star-invariant or model subspace (due to its role in yet another model - that of B. Sz.-Nagy and C. Foias).

Let $H^{2}$ denote the standard Hardy space in the unit disk, and let $\theta$ be an inner function in the disk. The model (or star-invariant) subspace $K_{\theta}$ of $H^{2}$ is then defined as

$$
K_{\theta}=H^{2} \ominus \theta H^{2} .
$$

According to the famous Beurling theorem, any closed subspace of $H^{2}$ invariant with respect to the backward shift in $H^{2}$ is of the form $K_{\theta}$. These subspaces play a distinguished role in operator theory (see, e.g., [11, 12]) and in operator-related complex analysis.

The details on the functional model for rank one perturbations will be given in the next section. For the moment, let us mention only that in this model the family of the eigenvectors of a rank one perturbation have a very transparent analytic meaning: they are either the families of reproducing kernels of $K_{\theta}$ or their biorthogonals.

Now we state our main result which says that there exist model spaces with a certain continuous family of vectors analogous to the properties of the vectors in Theorem 1.2. In view of the functional model (see Theorem 2.1) and Theorem 1.2 this immediately implies that there exists a unitary operator which has a hypercyclic rank one perturbation.

Theorem 1.3. There exists an inner function $\theta$ in the disk such that $\theta(0) \neq 0$ and $\theta$ is analytically continuable across some nonempty open subarc of $\mathbb{T}$, a function $\varphi \in H^{2} \backslash K_{\theta}$ and a sequence $\lambda_{n} \in \mathbb{T}$ such that the functions

$$
f_{n}(z)=\frac{\varphi(z)}{z-\lambda_{n}} \in K_{\theta}
$$

the family $\left\{f_{n}\right\}$ is complete in $K_{\theta}$ and, for any $n \geq 1$ and any $\varepsilon>0$, there exists $m \neq n$ such that $\left\|f_{n}-f_{m}\right\|<\varepsilon$.

We do not by any means claim that our proof is essentially shorter than the original proof by Grivaux. However, we believe that the application of the model clarifies the construction of the eigenvectors, since in the model space they have a special analytic structure: they are necessarily of the form (11) for some $\varphi \in H^{2}$.

As the original proof from [8], our construction is also inductive. However, the parameters are chosen in a different way. In particular, the eigenvalues of the operator $U+R$ will be chosen to be zeros of some Herglotz function (interlacing with the spectrum of $U$ ). The properties of Herglotz functions will play an important role in the construction. 


\section{Preliminaries on the FUnCtional MOdel FOR RANK-ONE PERTURBations OF UNITARY OPERATORS}

2.1. Inner functions and Clark measures. Recall that a function $\theta$ is said to be inner if it is analytic and bounded in the unit disk $\mathbb{D}$ and its nontangential boundary values satisfy $|\theta|=1$ a.e. with respect to the normalized Lebesgue measure $m$ on the unit circle $\mathbb{T}$.

Let $H^{2}=H^{2}(\mathbb{D})$ denote the Hardy space of the unit disk $\mathbb{D}$, equipped with the standard norm $\|\cdot\|_{2}=\|\cdot\|_{L^{2}(m)}$. With each inner function $\theta$ we associate the model subspace $K_{\theta}=H^{2} \ominus \theta H^{2}$.

The reproducing kernel for $K_{\theta}$ corresponding to a point $\lambda \in \mathbb{D}$ is given by

$$
k_{\lambda}(z)=\frac{1-\overline{\theta(\lambda)} \theta(z)}{1-\bar{\lambda} z} .
$$

Since functions in $K_{\theta}$ have more analyticity than general $H^{2}$ functions, there may exist reproducing kernels at boundary points. In particular, one can consider $k_{\lambda}, \lambda \in I$, if $\theta$ (and, hence, any function in $K_{\theta}$ ) have an analytic continuation across the arc $I$. More generally, by the results of Ahern and Clark we have $k_{\lambda} \in K_{\theta}$ for $\lambda \in \mathbb{T}$ if and only if $\left|\theta^{\prime}(\lambda)\right|<\infty$, the modulus of the angular derivative is finite.

Now we turn to Clark's construction of orthogonal bases of reproducing kernels [4]. For each $\alpha \in \mathbb{T}$, the function $\frac{\alpha+\theta}{\alpha-\theta}$ has positive real part in $\mathbb{D}$, and so there exists a finite (singular) positive measure $\mu^{\alpha}$ on $\mathbb{T}$ such that

$$
\operatorname{Re} \frac{\alpha+\theta(z)}{\alpha-\theta(z)}=\frac{1}{\pi} \int_{\mathbb{T}} \frac{1-|z|^{2}}{|\tau-z|^{2}} d \mu^{\alpha}(\tau), \quad z \in \mathbb{D} .
$$

Clark's theorem states that if, for some $\alpha, \mu^{\alpha}$ is purely atomic, i.e., if $\mu^{\alpha}=\sum_{n} \mu_{n} \delta_{\tau_{n}}$, $\tau_{n} \in \mathbb{T}$, then $k_{\tau_{n}} \in K_{\theta}$ and the system $\left\{k_{\tau_{n}}\right\}$ is an orthogonal basis in $K_{\theta}$. Note also that $\left\|k_{\tau_{n}}\right\|_{2}^{2}=\left|\theta^{\prime}\left(t_{n}\right)\right|=2 \mu_{n}^{-1}$.

Let $\mu=\mu^{1}$ be the Clark measure corresponding to $\alpha=1$. For $c \in L^{2}(\mu)$, put

$$
(V c)(z)=(1-\theta(z)) \int_{\mathbb{T}} \frac{c(\tau) d \mu(\tau)}{1-\bar{\tau} z}, \quad z \in \mathbb{D} .
$$

As Clark [4] has shown, $V$ is a unitary operator from $L^{2}(\mu)$ onto $K_{\theta}$. Moreover, the nontangential boundary values of the function $V c$ exist and coincide with $c \mu$-a.e. [13]. In particular, if $\left\{k_{\tau_{n}}\right\}$ is an orthogonal basis of reproducing kernels in $K_{\theta}$, then any $f \in K_{\theta}$ is of the form $f(z)=(1-\theta(z)) \sum_{n} \frac{c_{n} \mu_{n}}{1-\bar{\tau}_{n} z}$, where $\sum_{n}\left|c_{n}\right|^{2} \mu_{n}<\infty$.

2.2. The functional model. Now we give the details of the functional model for rank one perturbations of unitary operators. Let $\theta$ be an inner function in the disk such that $\theta(0) \neq 0$. For a function $f \in K_{\theta}$, the function $z f$ is not necessarily in $K_{\theta}$, but it is easily seen that $z f=\gamma+h$, where $\gamma \in \mathbb{C}$ is a constant and $h \in K_{\theta}$, and such decomposition is unique (moreover, $\gamma$ is a continuous functional of $f$ ). Now let $\varphi \in H^{2}$ be a function such that

$$
\varphi \notin K_{\theta}, \quad \frac{\varphi(z)-\varphi(0)}{z} \in K_{\theta} .
$$

Then we may define the operator $T=T_{\theta, \varphi}$ on $K_{\theta}$ by the formula

$$
T f:=z f-\gamma_{f} \varphi
$$


where $\gamma_{f}$ is the unique complex number such that $z f-\gamma_{f} \varphi \in K_{\theta}$.

We are ready to present the functional model of rank one perturbations which is analogous to [1, Theorem 0.6] (though the main ideas go back to [10]). Recall that a unitary operator is said to be singular if its spectral measure is singular with respect to the Lebesgue measure on $\mathbb{T}$. We also assume below that $U$ is cyclic, and so, up to a unitary equivalence, it is the operator of multiplication by $z$ in some space $L^{2}(\nu)$, where $\nu$ is a finite Borel measure on $\mathbb{T}$. By $\sigma(U)$ we denote the spectrum of $U$. Finally, we denote by $\rho(\theta)$ the boundary spectrum of an inner function $\theta$, that is, the complement of the union of all open $\operatorname{arcs} I$ such that $\theta$ admits an analytic continuation across $I$.

Theorem 2.1 (functional model). Let $U$ be a cyclic singular unitary operator such that $\sigma(U) \neq \mathbb{T}$. Then for any rank one perturbation $U+R$ of $U$ there exist an inner function $\theta$ in the disk such that $\theta(0) \neq 0$ and $\rho(\theta) \neq \mathbb{T}$, and a function $\varphi \in H^{2}$ satisfying (3) such that $U+R$ is unitary equivalent to the operator $T$ defined by (4).

Conversely, any inner function $\theta$ such that $\theta(0) \neq 0$ and $\rho(\theta) \neq \mathbb{T}$, and any function $\varphi \in H^{2}$ satisfying (3) correspond to some rank one perturbation $U+R$ of a cyclic singular unitary operator $U$ with $\sigma(U) \neq \mathbb{T}$.

It is obvious from the definition of the operator $T$ that if $\lambda \in \mathbb{C}$ is an eigenvalue of $T$, then the corresponding eigenvector is given by $\frac{\varphi(z)}{z-\lambda}$. Now, combining Theorems 2.1 and 1.2 we see that the existence of a rank one perturbation follows from Theorem 1.3 (since the functions $f_{n}$ are eigenvectors of some rank one perturbations).

2.3. Model spaces in the upper half-plane. It will be more convenient to work with model spaces in the half-plane $\mathbb{C}_{+}$rather than in the disk. We can translate our problem to the half-plane setting since the map $\tilde{f}(z)=\frac{1}{z+i} \cdot f\left(\frac{z-i}{z+i}\right)$ maps $H^{2}(\mathbb{D})$ to $H^{2}\left(\mathbb{C}_{+}\right)$and the model space $K_{\theta}$ in the disk to the model space $K_{\tilde{\theta}}=H^{2}\left(\mathbb{C}_{+}\right) \ominus \tilde{\theta} H^{2}\left(\mathbb{C}_{+}\right)$in $\mathbb{C}_{+}$, where $\tilde{\theta}(z)=\theta\left(\frac{z-i}{z+i}\right)$.

The definition and the properties of the Clark measures for the model spaces in the upper half-plane are analogous to those in the disk. Indeed, if $\theta$ is an inner function in $\mathbb{C}_{+}$, then the function $\frac{\alpha+\theta}{\alpha-\theta}$ has positive real part in $\mathbb{C}_{+}$, the measure $\mu$ on $\mathbb{R}$ from its Herglotz representation is said to be a Clark measure for $K_{\theta}$, and, again, the embedding of $K_{\theta}$ into $L^{2}(\mu)$ is a unitary operator (with one possible exception where a linear term appears in the Herglotz representation; we will exclude this case in our construction).

In particular, if the Clark measure corresponding to $\alpha=1$ is purely atomic, $\mu=$ $\sum_{n} \mu_{n} \delta_{t_{n}}$, then any function $f \in K_{\theta}$ is of the form

$$
f(z)=(1-\theta(z)) \sum_{n} \frac{c_{n} \mu_{n}}{z-t_{n}}, \quad \sum_{n}\left|c_{n}\right|^{2} \mu_{n}<\infty,
$$

and

$$
\|f\|_{L^{2}(\mathbb{R})}^{2}=4 \pi \sum_{n}\left|c_{n}\right|^{2} \mu_{n}=\pi \sum_{n}\left|f\left(t_{n}\right)\right|^{2} \mu_{n} .
$$

We will often use this formula for the norm in what follows. 


\section{Proof of Theorem 1.3}

3.1. Plan of the solution. By the above functional model, the problem is reduced to the construction of functions $\theta$ and $\varphi$ as in Theorem 1.3. As explained in Subsection [2.3. we can work on the real line and in the upper half-plane. Thus, in what follows $\|\cdot\|_{2}$ stands for the usual $L^{2}$-norm on $\mathbb{R}$.

We will construct:

- a countable set $T=\left\{t_{n}\right\}_{n=1}^{\infty}$ on some interval (say, in $[0,1]$ );

- a finite measure $\mu=\sum_{n=1}^{\infty} \mu_{n} \delta_{t_{n}}$;

- an inner function $\theta$ defined by

$$
\frac{1+\theta(z)}{1-\theta(z)}:=i \sum_{n=1}^{\infty} \frac{\mu_{n}}{z-t_{n}}
$$

- a function $\varphi$ of the form

$$
\varphi(z):=(1-\theta(z))\left[\sum_{n=1}^{\infty} \frac{c_{n} \mu_{n}}{t_{n}-z}+1\right], \quad \sum_{n=1}^{\infty}\left|c_{n}\right|^{2} \mu_{n}<\infty,
$$

such that for some sequence $\left\{\lambda_{j}\right\} \subset[0,1]$, we have $\frac{\varphi}{z-\lambda_{j}} \in L^{2}(\mathbb{R})$ and, for any $j \geq 1$ and $\varepsilon>0$ there exists $k \neq j$ such that

$$
\left\|\frac{\varphi(z)}{z-\lambda_{j}}-\frac{\varphi(z)}{z-\lambda_{k}}\right\|_{2}<\varepsilon .
$$

Note that in this construction $\mu$ is a Clark measure for $\theta$.

As in Grivaux' paper [8] we will proceed with the construction inductively. Namely, on the $N$-th step we construct $t_{1}, \ldots, t_{N} \in[0,1], \mu_{1}, \ldots, \mu_{N}$ and functions $\theta_{N}$ defined by

$$
i \frac{1+\theta_{N}(z)}{1-\theta_{N}(z)}:=\sum_{n=1}^{N} \frac{\mu_{n}}{t_{n}-z}
$$

and $\varphi_{N}$

$$
\varphi_{N}(z):=\left(1-\theta_{N}(z)\right)\left[1+\sum_{n=1}^{N} \frac{c_{n} \mu_{n}}{t_{n}-z}\right]
$$

where $c_{n}>0$. The sequences $\mu_{n}$ and $c_{n}$ will be assumed to tend to zero very rapidly.

It is a key idea of the construction that $c_{n}$ are taken to be positive. In this case the function $1+\sum_{n=1}^{N} \frac{c_{n} \mu_{n}}{t_{n}-z}$ (which appears in the definition of $\varphi_{N}$ ) is a Herglotz function (has positive real part in $\mathbb{C}_{+}$) and, therefore, it has exactly $N$ zeros $\lambda_{1}^{N}, \lambda_{2}^{N}, \ldots \lambda_{N}^{N}$, interlacing with the points $t_{1}, \ldots, t_{N}$. Choosing $c_{n}$ sufficiently small we can control the location of these points.

Note that $\theta_{N}$ is a finite Blaschke product and the model space $K_{\theta_{N}}$ is $N$-dimensional. The measure $\sum_{n=1}^{N} \mu_{n} \delta_{t_{n}}$ is a Clark measure for $K_{\theta_{N}}$. Also, $\varphi_{N} \notin K_{\theta_{N}}$, since $1-\theta_{N} \notin$ $K_{\theta_{N}}$, while

$$
f_{j}^{N}(z):=\frac{\varphi_{N}(z)}{z-\lambda_{j}^{N}} \in K_{\theta_{N}} .
$$


Indeed, we have a representation of type (51),

$$
f_{j}^{N}(z)=\frac{\varphi_{N}(z)-\varphi_{N}\left(\lambda_{j}^{N}\right)}{z-\lambda_{j}^{N}}=\left(1-\theta_{N}(z)\right) \sum_{n=1}^{N} \frac{c_{n} \mu_{n}}{\left(\lambda_{j}^{N}-t_{n}\right)\left(z-t_{n}\right)} .
$$

Assume that $t_{n}, \mu_{n}$ and $c_{n}, 1 \leq n \leq N_{1}$, are already chosen. On $N$-th step we will add a point $t_{N} \in(0,1)$, its mass $\mu_{N}$ and a coefficient $c_{N}$ in the following order. First, we take $t_{N}$ to be very close to some zero of the function $\varphi_{N-1}$. Then we choose $\mu_{N}$ to be so small that $\theta_{N}$ does not differ much from $\theta_{N-1}$ outside a small neighborhood of $t_{N}$. Finally, we choose $c_{N}$ to be even much smaller than $\mu_{N}$ so that all zeros $\lambda_{1}^{N}, \ldots \lambda_{N}^{N}$ from generation $N$ almost coincide with the corresponding zeros $\lambda_{1}^{N-1}, \ldots \lambda_{N-1}^{N-1}$ from generation $N-1$, while the zero $\lambda_{N}^{N}$ is very close to $t_{N}$.

Let us formally state what we need for the convergence.

(I) We will choose $\mu_{n}>0$ and $c_{n}>0$ so that $\mu_{n}<2^{-n}$ and $c_{n}<2^{-n}$. These conditions already ensure the convergence of the functions (10) and (11) to (7) and (8) respectively. Also, we will require that $\left|\lambda_{j}^{N-1}-\lambda_{j}^{N}\right|<2^{-N}, j=1, \ldots, N-1$ (in fact we will need much more, see (18) below).

(II) Clearly, for each $N$, the functions $f_{j}^{N}, j=1, \ldots, N$, are linearly independent, and so they form a basis in $K_{\theta_{N}}$. Let $A_{N}$ be a sort of a $\ell^{1}$ basis constant for $\left\{f_{j}^{N}\right\}$ : for any $\left\{\alpha_{j}\right\}_{j=1}^{N}, \alpha_{j} \in \mathbb{C}$,

$$
\sum_{j=1}^{N}\left|\alpha_{j}\right| \leq A_{N}\left\|\sum_{j=1}^{N} \alpha_{j} f_{j}^{N}\right\|_{2}
$$

Without loss of generality we assume that $A_{N} \geq 1$ and the sequence $A_{N}$ increases. Our second requirement then reads as follows:

$$
\left\|f_{j}^{N}-f_{j}^{N-1}\right\|_{2}<\frac{1}{2^{N+2} A_{N-1}}, \quad j=1, \ldots, N-1 .
$$

(III) Let $l(n)$ be a sequence of integers such that $l(n)<n$ and $l(n)$ takes every integer value infinitely many times (e.g., $1,1,2,1,2,3,1,2,3,4, \ldots)$. To achieve property (9), we will introduce the third requirement:

$$
\left\|f_{l(N)}^{N}-f_{N}^{N}\right\|_{2}<2^{-N-1}, \quad N \in \mathbb{N}
$$

3.2. Choice of the parameters. Assume that $t_{n}, \mu_{n}$ and $c_{n}, n=1, \ldots, N-1$, are already chosen. First we choose the point $t_{N}$. Let $\varepsilon_{N}$ be some small positive number (namely, let $\varepsilon_{N} \leq 4^{-N-2} A_{N-1}^{-1}$, where $A_{N-1}$ is the constant from (II) above) and consider the equation

$$
1+\sum_{n=1}^{N-1} \frac{c_{n} \mu_{n}}{t_{n}-x}=\varepsilon_{N}
$$

Clearly, it has $N-1$ real zeros which depend continuously on $\varepsilon_{N}$. Hence, they can be enumerated $x_{1}, \ldots x_{N-1}$ in such a way that $x_{j} \rightarrow \lambda_{j}^{N-1}$ when $\varepsilon_{N} \rightarrow 0$. 
Let us take $\varepsilon_{N}$ to be so small that if we take as $t_{N}$ the zero of (15) which is the closest to the point $\lambda_{l(N)}^{N-1}$, then

$$
\left|t_{N}-\lambda_{l(N)}^{N-1}\right|<4^{-3 N} \delta_{N-1}^{3}
$$

where

$$
\delta_{N-1}=\inf _{1 \leq j, n \leq N-1}\left|\lambda_{j}^{N-1}-t_{n}\right| .
$$

Now we put $\mu_{N}=\sqrt{\varepsilon_{N}}$ and define the inner function $\theta_{N}$ by (10). One more additional restriction on the smallness of $\varepsilon_{N}$ will be as follows:

$$
\left\|\frac{\theta_{N}(z)-\theta_{N-1}(z)}{z-t_{n}}\right\|_{2}<\frac{1}{4^{N} A_{N-1}}, \quad n=1, \ldots, N-1 .
$$

This is also possible by continuous dependence of these norms from $\varepsilon_{N}$. More precisely, by the construction of $t_{N}$ we have $t_{N} \rightarrow \lambda_{l(N)}^{N-1}$ as $\varepsilon_{N} \rightarrow 0$, and so $t_{N}$ is separated from $t_{n}, 1 \leq n \leq N-1$. Therefore, we can choose a small neighborhood $I$ of $\lambda_{l(N)}^{N-1}$ such that the integral of $\left|\frac{\theta_{N}(t)-\theta_{N-1}(t)}{t-t_{n}}\right|^{2}$ over $I$ is small. Once the interval $I$ is fixed, we can make the integral over $\mathbb{R} \backslash I$ to be arbitrarily small since $\theta_{N}-\theta_{N-1}$ (as well as $\theta_{N}^{\prime}-\theta_{N-1}^{\prime}$ ) tends to zero uniformly over $\mathbb{R} \backslash I$ as $\varepsilon_{N} \rightarrow 0$.

Finally, let us choose $c_{N}$. Consider the equation $\varphi_{N}(x)=0$ which is equivalent to

$$
1+\sum_{n=1}^{N-1} \frac{c_{n} \mu_{n}}{t_{n}-x}+\frac{c_{N} \mu_{N}}{t_{N}-x}=0 .
$$

Again, a continuity argument shows that we may enumerate the $N$ zeros of this equation $\lambda_{j}^{N}$ (which should be thought of as functions of $c_{N}$ ) so that $\lambda_{j}^{N} \rightarrow \lambda_{j}^{N-1}, j=1, \ldots, N-1$, and $\lambda_{N}^{N} \rightarrow t_{N}$ as $c_{N} \rightarrow 0$. Moreover, by the choice of $t_{N}$ as a solution of (15), we have

$$
1+\sum_{n=1}^{N-1} \frac{c_{n} \mu_{n}}{t_{n}-\lambda_{N}^{N}} \rightarrow \varepsilon_{N}
$$

and $\lambda_{N}^{N} \rightarrow t_{N}$ as $c_{N} \rightarrow 0$. Therefore,

$$
\lambda_{N}^{N}-t_{N} \sim \frac{c_{N} \mu_{N}}{\varepsilon_{N}}=\frac{c_{N}}{\sqrt{\varepsilon_{N}}}, \quad c_{N} \rightarrow 0 .
$$

Let us now choose $c_{N}$ to be so small that

$$
\begin{gathered}
\left|\lambda_{j}^{N}-\lambda_{j}^{N-1}\right|<\frac{1}{4^{3 N} A_{N-1}} \delta_{N-1}^{3}, \quad j=1, \ldots, N-1, \\
\frac{c_{N}}{2 \sqrt{\varepsilon_{N}}} \leq\left|\lambda_{N}^{N}-t_{N}\right|<\left|\lambda_{l(N)}^{N}-t_{N}\right|,
\end{gathered}
$$

and

$$
\left|\lambda_{N}^{N}-\lambda_{l(N)}^{N-1}\right|<2^{-N} \delta_{N-1}^{3} .
$$

The latter estimate is possible by (16) and (18). 
3.3. Proof of (13) . To estimate the norm $\left\|f_{j}^{N}-f_{j}^{N-1}\right\|_{2}, 1 \leq j \leq N-1$, we write, using (12),

$$
\begin{aligned}
\frac{\varphi_{N-1}(z)}{z-\lambda_{j}^{N-1}} & -\frac{\varphi_{N}(z)}{z-\lambda_{j}^{N}}=\sum_{n=1}^{N-1} \frac{c_{n} \mu_{n}\left(1-\theta_{N-1}(z)\right)}{\left(t_{n}-\lambda_{j}^{N-1}\right)\left(z-t_{n}\right)} \\
& -\sum_{n=1}^{N-1} \frac{c_{n} \mu_{n}\left(1-\theta_{N}\right)}{\left(t_{n}-\lambda_{j}^{N}\right)\left(z-t_{n}\right)}-\frac{c_{N} \mu_{N}\left(1-\theta_{N}\right)}{\left(t_{N}-\lambda_{j}^{N}\right)\left(z-t_{N}\right)} .
\end{aligned}
$$

Denote the last summand in (21) by $h$. Then $h \in K_{\theta_{N}}$ (it is a reproducing kernel up to a coefficient), and so

$$
\|h\|_{2}^{2}=\frac{\left|c_{N}\right|^{2} \mu_{N}}{\left|t_{N}-\lambda_{N}^{N}\right|^{2}} \leq 4 \mu_{N} \varepsilon_{N} .
$$

The first two summands in (21) may be rearranged into the sum of

$$
g_{1}(z)=\left(1-\theta_{N}(z)\right) \sum_{n=1}^{N-1}\left(\frac{c_{n} \mu_{n}}{\left(t_{n}-\lambda_{j}^{N-1}\right)\left(z-t_{n}\right)}-\frac{c_{n} \mu_{n}}{\left(t_{n}-\lambda_{j}^{N}\right)\left(z-t_{n}\right)}\right)
$$

and

$$
g_{2}(z)=\sum_{n=1}^{N-1} c_{n} \mu_{n} \frac{\theta_{N}(z)-\theta_{N-1}(z)}{\left(t_{n}-\lambda_{j}^{N-1}\right)\left(z-t_{n}\right)} .
$$

We may compute the norm of $g_{1} \in K_{\theta_{N}}$ using Clark's theorem (see formula (6) ):

$$
\left\|g_{1}\right\|_{2}^{2}=\sum_{m=1}^{N-1}\left|g_{1}\left(t_{m}\right)\right|^{2} \mu_{m}=\sum_{m=1}^{N-1} \frac{\left|\lambda_{j}^{N}-\lambda_{j}^{N-1}\right|^{2}\left|c_{m}\right|^{2} \mu_{m}}{\left|t_{m}-\lambda_{j}^{N-1}\right|^{2}\left|t_{m}-\lambda_{j}^{N}\right|^{2}} .
$$

Thus, $\left\|g_{1}\right\|_{2}$ does not exceed $4^{-N} A_{N-1}^{-1}$ by (18) .

Finally, $\left\|g_{2}\right\|_{2} \leq 4^{-N} A_{N-1}^{-1}$, and summing the above estimates we obtain (13).

3.4. Proof of (14). To estimate the norm $\left\|f_{l(N)}^{N}-f_{N}^{N}\right\|_{2}$ we use again Clark's theorem:

$$
\left\|\frac{\varphi_{N}}{z-\lambda_{l(N)}^{N}}-\frac{\varphi_{N}}{z-\lambda_{N}^{N}}\right\|_{2}^{2}=\sum_{m=1}^{N}\left|\frac{\varphi_{N}\left(t_{m}\right)}{t_{m}-\lambda_{l(N)}^{N}}-\frac{\varphi_{N}\left(t_{m}\right)}{t_{m}-\lambda_{N}^{N}}\right|^{2} \mu_{m} .
$$

Let $L_{N}=\left|\lambda_{l(N)}^{N}-\lambda_{N}^{N}\right|$. Then, by (18) and (20) $),\left|t_{m}-\lambda_{j}^{N}\right|>L_{N}^{1 / 3}$ for any $m=1 \ldots N-1$ and $j=1, \ldots, N$. Now the first $N-1$ summands in (22) may be estimated as

$$
\sum_{m=1}^{N-1} \frac{\left|\lambda_{l(N)}^{N}-\lambda_{N}^{N}\right|^{2}}{\left|t_{m}-\lambda_{l(N)}^{N}\right|^{2}\left|t_{m}-\lambda_{N}^{N}\right|^{2}}\left|\varphi_{N}\left(t_{m}\right)\right|^{2} \mu_{m} \leq \sum_{m=1}^{N-1} L_{N}^{2 / 3}\left|\varphi_{N}\left(t_{m}\right)\right|^{2} \mu_{m} .
$$

Since by (18) and (20) $L_{N} \leq 2^{-3 N} \delta_{N-1}^{3}$ and $\left|\varphi_{N}\left(t_{m}\right)\right|=\left|\theta_{N}^{\prime}\left(t_{m}\right)\right| c_{m} \mu_{m}=2 c_{m}$, we conclude that the latter sum does not exceed $2^{-N-1}$.

It remains to consider the summand with the number $N$. We have

$$
\left|\frac{\varphi_{N}\left(t_{N}\right)}{t_{N}-\lambda_{N}^{N}}\right|=\frac{2}{\mu_{N}} \cdot \frac{c_{N} \mu_{N}}{\left|t_{N}-\lambda_{N}^{N}\right|} \leq \frac{2 \varepsilon_{N}}{\mu_{N}}=2 \sqrt{\varepsilon_{N}} \leq 2^{-N-2} .
$$

Here we used inequality (19). The estimate for the term $\left|\frac{\varphi_{N}}{t_{N}-\lambda_{l(N)}^{N}}\right|$ is analogous. Estimate (14) is proved 
3.5. Convergence and completeness. To ensure the pointwise convergences $\theta_{N}(z) \rightarrow \theta(z)$ and $\varphi_{N}(z) \rightarrow \varphi(z), z \in \mathbb{C}_{+}$, it suffices to assume only that $\sum_{n} \mu_{n}<\infty$ and $c_{n} \rightarrow 0$.

By the choice of the parameters in Subsection [3.2, the sequence $\lambda_{j}^{N}$ converges to some point $\lambda_{j}$, and it follows that $f_{j}^{N}$ converges to $\frac{\varphi(z)}{z-\lambda_{j}}$ pointwise in $\mathbb{C}_{+}$. On the other hand, by (13), the sequence $f_{j}^{N} \in H^{2}$ converges to some function $f_{j}$ in $H^{2}$ (recall that $H^{2}=H^{2}\left(\mathbb{C}_{+}\right)$is the Hardy space in the upper half-plane). Since the convergence in $H^{2}$ implies the pointwise convergence in $\mathbb{C}_{+}$, we conclude that $f_{j}(z)=\frac{\varphi(z)}{z-\lambda_{j}}$.

Next we prove that the family $\left\{f_{j}\right\}$ is complete in $K_{\theta}$. First of all, note that if $g \in K_{\theta}$,

$$
g(z)=(1-\theta(z)) \sum_{n=1}^{\infty} \frac{d_{n} \mu_{n}}{z-t_{n}}, \quad g_{N}(z)=\left(1-\theta_{N}(z)\right) \sum_{n=1}^{N} \frac{d_{n} \mu_{n}}{z-t_{n}}
$$

then $\left\|g-g_{N}\right\|_{2} \rightarrow 0, N \rightarrow \infty$. Indeed,

$$
g(z)-g_{N}(z)=\left(\theta_{N}(z)-\theta(z)\right) \sum_{n=1}^{N} \frac{d_{n} \mu_{n}}{z-t_{n}}+(1-\theta(z)) \sum_{n=N+1}^{\infty} \frac{d_{n}}{z-t_{n}} .
$$

The norm of the second sum by Clark's theorem (see ([6]) ) equals $\sum_{m=N+1}^{\infty}\left|d_{m}\right|^{2} \mu_{m}$, which obviously goes to zero, $N \rightarrow \infty$, while the norm of the first sum is small by the assumption (17).

Thus, we constructed a sequence $g_{N} \in K_{\theta_{N}}$ such that $g_{N} \rightarrow g$ in $L^{2}(\mathbb{R})$. It remains to approximate functions $g_{N}$ by linear combinations of $f_{j}$. The method is borrowed from [8]. Since $\left\{f_{j}^{N}\right\}$ is a basis in $K_{\theta_{N}}$, we may write $g_{N}=\sum_{j=1}^{N} \alpha_{j} f_{j}^{N}$. Then, making use of (13), we get

$$
\begin{aligned}
\left\|g-\sum_{j=1}^{N} \alpha_{j} f_{j}\right\|_{2} & \leq \sum_{j=1}^{N}\left|\alpha_{j}\right| \sum_{k=N}^{\infty}\left\|f_{j}^{k}-f_{j}^{k+1}\right\|_{2} \\
& \leq \frac{1}{2^{N} A_{N}} \sum_{j=1}^{N}\left|\alpha_{j}\right| \leq 2^{-N}\left\|g_{N}\right\|_{2},
\end{aligned}
$$

which goes to 0 as $N \rightarrow \infty$. Completeness of the family $\left\{f_{j}\right\}$ is proved.

3.6. End of the proof of Theorem 1.3. Let us complete the proof of Theorem 1.3. We have constructed a complete sequence $\left\{f_{j}\right\}=\left\{\frac{\varphi}{z-\lambda_{j}}\right\}$ in $K_{\theta}$. It remains to verify that $\left\{f_{j}\right\}$ has the property (9). Let $j \geq 1$ and $\varepsilon$ be given. Choose $N$ such that $l(N)=j$ and $2^{-N}<\varepsilon$, which is possible by the definition of the sequence $l(n)$. Then, by (14), $\left\|f_{j}^{N}-f_{N}^{N}\right\|_{2}<2^{-N-1}$. Also, by (14),

$$
\left\|f_{N}^{N}-f_{N}\right\|_{2} \leq \sum_{k=N}^{\infty}\left\|f_{N}^{k}-f_{N}^{k+1}\right\|_{2} \leq 2^{-N-2}
$$

and, analogously, $\left\|f_{j}^{N}-f_{j}\right\|_{2} \leq 2^{-N-1}$. Combining these estimates we obtain $\| f_{j}-$ $f_{N} \|_{2} \leq 2^{-N}$. 


\section{Concluding Remarks}

The unitary operator $U$ in our construction (as well as in [8]) is of a very special form. Note that if $t_{n} \in \mathbb{R}$ is the sequence constructed in Section 3, then the spectrum of $U$ is given by $\left\{\tau_{n}\right\}, \tau_{n}=\frac{t_{n}-i}{t_{n}+i}$. The points $t_{n}$ are chosen inductively so that $t_{N}$ be close to some of the points $t_{n}, 1 \leq n \leq N-1$, and so the set $\left\{t_{n}\right\}$ has a certain self-similarity. It seems to be a natural question, which unitary operators have hypercyclic rank one perturbations.

Problem 1. To describe cyclic unitary operators $U$ such that $U+R$ is hypercyclic for some rank one operator $R$.

In particular, it is not clear whether the spectrum $\sigma(U)$ can have nonempty interiour or positive measure.

Problem 2. Does there exist a cyclic unitary operator $U$ such that $U+R$ is hypercyclic for some rank one operator $R$ and $\sigma(U)=\mathbb{T}$.

As in [8], in the present paper the spectral measure of $U$ is purely atomic.

Problem 3. Construct a unitary operator $U$, whose spectral measure is an absolutely continuous or a continous (i.e., without point masses) singular measure on $\mathbb{T}$, such that $U+R$ is hypercyclic for some rank one operator $R$.

Note that the functional model applies to rank one perturbations of an arbitrary cyclic unitary operator whose spectral measure is singular. Therefore, one can hope to obtain further information about hypercyclic rank one perturbations of unitary operators using this model.

\section{REFERENCES}

[1] A. Baranov, D. Yakubovich, Completeness and spectral synthesis of nonselfadjoint onedimensional perturbations of selfadjoint operators, arXiv:1212.5965.

[2] F. Bayart, E. Matheron, Dynamics of Linear Operators, Cambridge University Press (2009).

[3] K.C. Chan, J.H. Shapiro, The cyclic behavior of translation operators on Hilbert spaces of entire functions, Indiana Math. J. 40, 4 (1991), 1421-1449.

[4] D.N. Clark, One-dimensional perturbations of restricted shifts, J. Anal. Math. 25 (1972), 169-191.

[5] R.M. Gethner, J.H. Shapiro, Universal vectors for operators on spaces of holomorphic functions, Proc. Amer. Math. Soc. 100 (1987), 281-288.

[6] G. Godefroy, J.H. Shapiro, Operators with dense, invariant cyclic vector manifolds, J. Funct. Anal. 98 (1991), 229-269.

[7] S. Grivaux, A new class of frequently hypercyclic operators, Indiana Univ. Math. J. 60 (2011), $1177-1201$.

[8] S. Grivaux, A hypercyclic rank one perturbation of a unitary operator, Math. Nachr. 285 (2012), $5-6,533-544$.

[9] K.-G. Grosse-Erdmann, A. Peris Manguillot, Linear Chaos, Universitext, Springer, London, 2011.

[10] V.V. Kapustin, One-dimensional perturbations of singular unitary operators, Zapiski Nauchn. Sem. POMI 232 (1996), 118-122; English transl. in J. Math. Sci. (New York) 92 (1998), 1, 3619-3621.

[11] N.K. Nikol'skii, Treatise on the Shift Operator, Springer-Verlag, Berlin, 1986.

[12] N.K. Nikolski, Operators, Functions, and Systems: an Easy Reading, Math. Surveys Monogr., Vol. 92-93, AMS, Providence, RI, 2002.

[13] A.G. Poltoratski, Boundary behavior of pseudocontinuable functions, Algebra i Analiz 5 (1993), 2, 189-210; English transl. in St. Petersburg Math. J. 5 (1994), 2, 389-406.

[14] S. Shkarin, A hypercyclic finite rank perturbation of a unitary operator, Math. Ann. 348 (2010), 379-393. 
Anton Baranov,

Department of Mathematics and Mechanics, St. Petersburg State University, St. Petersburg, Russia, AND

National Research University Higher School of Economics, St. Petersburg, Russia,

anton.d.baranov@gmail.com

ANDREI LISHANSKII,

Department of Mathematics and Mechanics, St. Petersburg State University, St. Petersburg, Russia, AND

Chebyshev Laboratory, St. Petersburg State University, St. Petersburg, Russia, lishanskiyaa@gmail.com 